\title{
CÁSCARAS DE HUEVO DE TIPO Aepyornis DEL MIOCENO SUPERIOR DE ALICANTE (ESPAÑA). APROXIMACIÓN FILOGENÉTICA
}

\author{
Ana María BRAVO ${ }^{1}$, Alfonso YÉBENES ${ }^{2}$ y Hugo \\ MARTÍN ABAD \\ ${ }^{1}$ Unidad de Paleontología, Dpto. Biología, Facultad Ciencias, Universidad \\ Autónoma de Madrid, 28049 Cantoblanco, Madrid. E-mail: ana.bravo@estu- \\ diante.uam.es \\ ${ }^{2}$ Dpto. Ciencias de la Tierra y del Medio Ambiente, Universidad de Alicante, \\ Apdo. Correos, 99, 03080 Alicante.
}

Bravo, A. M., Yébenes, A. \& Martín Abad, H. 2009. Cáscaras de huevo de tipo Aepyornis del Mioceno Superior de Alicante (España). Aproximación filogenética. [Eggshells of Aepyornis type from Upper Miocene of Alicante (Spain). A phylogenetic approach.] Revista Española de Paleontología, 24 (1), 47-58. ISSN 0213-6937.

\begin{abstract}
The Torrellano site (Upper Miocene, Alicante Province, Spain) has yielded hundreds of eggshell fragments of the ornithoid basic type, characterised mainly by their important thickness and by the presence of pores inside slit-like grooves. Similar eggshells have been found in many Tertiary and Quaternary sites of Asia, Africa and Europe. In all cases it has been established the similarity with the eggshells of the extinct giant Aepyornithidae ratite Aepyornis maximus.

The eggshells of the new Torrellano site are described and compared with similar material. Some of the described characters are used in a cladistic analysis together with extant ratites (Struthio, Rhea, Apteryx, Dromaius, Casuarius), the genus Tinamus, and fossil material (Diamantornis wardi, Diamantornis corbetti and Dinornithidae). The resulting cladogram from extant ratite data, corroborates previous works and show that eggshells characters bear phylogenetic information and thus are useful in the establishment of relationship hypothesis. Torrellano eggshells are included within the crown-group ratites, although in a politomy which can not be resolved with the used characters. On the other side, Struthio appears as the sister group of the parataxa $D$. wardi y $D$. corbetti, which could confirm previous hypothesis of their possible relationship with the family Struthionidae.
\end{abstract}

Keywords: Eggshells, ratites, Miocene, phylogenetic analysis, Aepyornis, Diamantornis.

\section{RESUMEN}

El yacimiento de Torrellano (Mioceno Superior, provincia de Alicante) ha proporcionado cientos de fragmentos de cáscara de tipo básico ornitoide, caracterizadas fundamentalmente por su gran grosor y por presentar los poros en el interior de hendiduras alargadas. Se han encontrado cáscaras de estructura similar en numerosos yacimientos del Terciario y Cuaternario de Asia, África y Europa. En todos los casos se ha establecido su semejanza con las cáscaras de la extinta ratite gigante Aepyornis maximus (Aepyornithidae).

Se describen las cáscaras del nuevo yacimiento de Torrellano y se comparan con material similar. Algunos de los caracteres descritos han sido utilizados para realizar un análisis cladístico junto con ratites actuales (Struthio, Rhea, Apteryx, Dromaius, Casuarius), el género Tinamus, y material fósil (Diamantornis wardi, Diamantornis corbetti y Dinornithidae). El cladograma obtenido a partir de los datos de ratites actuales corrobora trabajos previos y muestra que los caracteres de las cáscaras están relacionados con la filogenia y son, por tanto, útiles para establecer hipótesis de parentesco. En este caso, el material de Torrellano, queda incluido dentro del crown group de ratites, aunque formando parte de una politomía que no puede resolverse con los caracteres empleados. Por otro lado, Struthio aparece como grupo hermano de los parataxones $D$. wardi y D. corbetti, lo cual podría confirmar las hipótesis existentes sobre la posible relación de estos parataxones con la familia Struthionidae.

Palabras clave: Cáscaras, ratites, Mioceno, análisis filogenético, Aepyornis, Diamantornis. 


\section{INTRODUCCIÓN}

La especie de ave ratite Aepyornis maximus St. Hilaire, 1851 fue el representante de mayor tamaño de la familia Aepyornithidae que pobló la isla de Madagascar hasta tiempos recientes (Fuller, 2001). Además de elementos esqueléticos se han hallado fragmentos de cáscaras, huevos completos, e incluso parte de un embrión fosilizado en el interior de un huevo (Sauer, 1972; Balanoff, 2003). El registro de cáscaras similares a las de Aepyornis St. Hilaire, 1851 es relativamente abundante, con hallazgos en la Península Ibérica (Mein \& Dauphin, 1995), las islas Canarias (Sauer \& Rothe, 1972), Asia (Sauer, 1972; Stern et al., 1994) y África (Senut et al., 1995; Dauphin et al., 1996; Stidham, 2004; Bibi et al. 2006). En la mayoría de los casos los autores emplean el término "aepyornitoide" para indicar su similitud con el material de Madagascar, aunque este término también designa un tipo de sistema de canales (Hirsch et al., 1997). Con el fin de evitar confusiones, en este trabajo se utiliza el término "tipo Aepyornis" para indicar la semejanza entre el material de Torrellano y el de la especie Aepyornis maximus.

Las cáscaras y huevos fósiles son restos indirectos que proporcionan información filogenética, mostrando una estructura básica estable dentro de los principales taxones de amniotas (Mikhailov et al., 1996; Kohring, 1999). A partir de este tipo de fósiles se puede obtener además información sobre reproducción, cuidados parentales, comportamiento, paleoambientes e incluso, en ocasiones, han sido empleados en estudios biocronológicos (Stern et al., 1994; Horner, 2000; Garcia \& Vianey-Liaud, 2001; Segalen et al., 2002). Los estudios sobre cáscaras y huevos fósiles han sido tradicionalmente descriptivos y enfocados a su clasificación parataxonómica; sin embargo en los últimos años se han empleado los caracteres de las cáscaras en la realización de análisis cladísticos quedando demostrada su utilidad filogenética (Grellet-Tinner, 2000; Zelenitsky \& Modesto, 2003; García et al., 2006). Siguiendo este tipo de aproximación, en el presente trabajo se ha llevado a cabo en primer lugar el estudio y descripción del material de Torrellano. Este nuevo yacimiento ha proporcionado varios cientos de fragmentos de cáscaras caracterizados básicamente por su relativo gran grosor y por la presencia de poros en el interior de surcos alargados, de modo similar a la de las cáscaras del género Aepyornis. En segundo lugar, se han empleado algunos de los caracteres micro- y macroestructurales descritos para la realización de un análisis cladístico. Este análisis ha sido doble: por un lado se han analizado los caracteres de las cáscaras de cinco géneros de ratites actuales (Struthio Linneo, 1758; Rhea Bonaparte, 1849; Aptery Shaw, 1813; Dromaius Vieilot, 1816; Casuarius Linneo, 1771), más Tinamus Huxley, 1872 como grupo externo; y por otro se analizaron conjuntamente aves actuales y cuatro tipos de cáscaras fósiles (dinornítidos, Diamantornis wardi Pickford, Senut \& Dauphin, 1995, Diamantornis corbetti Pickford Senut \& Dauphin, 1995, y Torrellano).

Para la realización del presente estudio no ha sido posible el acceso a material original de cáscaras de la especie Aepyornis maximus. Por otro lado, los datos bibliográficos sobre las cáscaras de dicha especie son escasos y se suelen limitar a medidas de grosor y descripciones sobre la morfología de los poros, no existiendo, por ejemplo, datos sobre proporciones entre capas, estructura de la zona externa (EZ) o de la capa mamilar. Debido a esto, no se han incluido caracteres de las cáscaras de dicha especie en nuestro análisis cladístico.

\section{CONTEXTO GEOLÓGICO}

El yacimiento de cáscaras de huevos de Torrellano ha sido localizado en materiales del Messiniense de la Sierra del Colmenar, en un punto situado entre las poblaciones de Elche y Alicante (Fig. 1a). La Sierra del Colmenar forma parte del borde norte de la Cuenca del Bajo Segura, una de las diferentes cuencas neógenas marginales del Mediterráneo occidental que afloran en la parte oriental de la Cordillera Bética.

La estratigrafía del borde norte de la Cuenca del Bajo Segura ha sido estudiada con detalle por Soria et al. (2003, 2005). Estos autores han dividido la sucesión sedimentaria del Messiniense y Plioceno en unidades aloestratigráficas limitadas por discontinuidades mayores. En el Messiniense establecen dos unidades (MI y MII) separadas por una discontinuidad denominada intramessiniense. Asimismo se considera una única unidad aloestratigráfica del Plioceno $(\mathrm{P})$, dentro de la que distinguen tres unidades litoestratigráficas (P1, P2 y P3), que está separada de la unidad MII por la discontinuidad fini-Messiniense. Cabe destacar que en la Sierra de Colmenar no existe la unidad litológica pliocena P1, ya que ésta sólo se depositó en antiguos paleovalles, inundados por la transgresión pliocena, como los de Elche y Crevillente.

Figura 1. a, Esquema de situación y mapa geológico simplificado del sector norte de la Cuenca del Bajo Segura con localización del yacimiento de cáscaras de huevos de tipo Aepyornis. b. Sección estratigráfica del Messiniense y Plioceno del sector de la Sierra del Colmenar donde se localiza el yacimiento.

$\boldsymbol{a}$, Scheme of the situation and simplified geological map of the north sector of the Bajo Segura Basin, with the location of the type Aepyornis eggshell site. $\boldsymbol{b}$, Stratigraphical section of the Messinian and Pliocene of the Sierra del Colmenar sector where the site is located. 
a
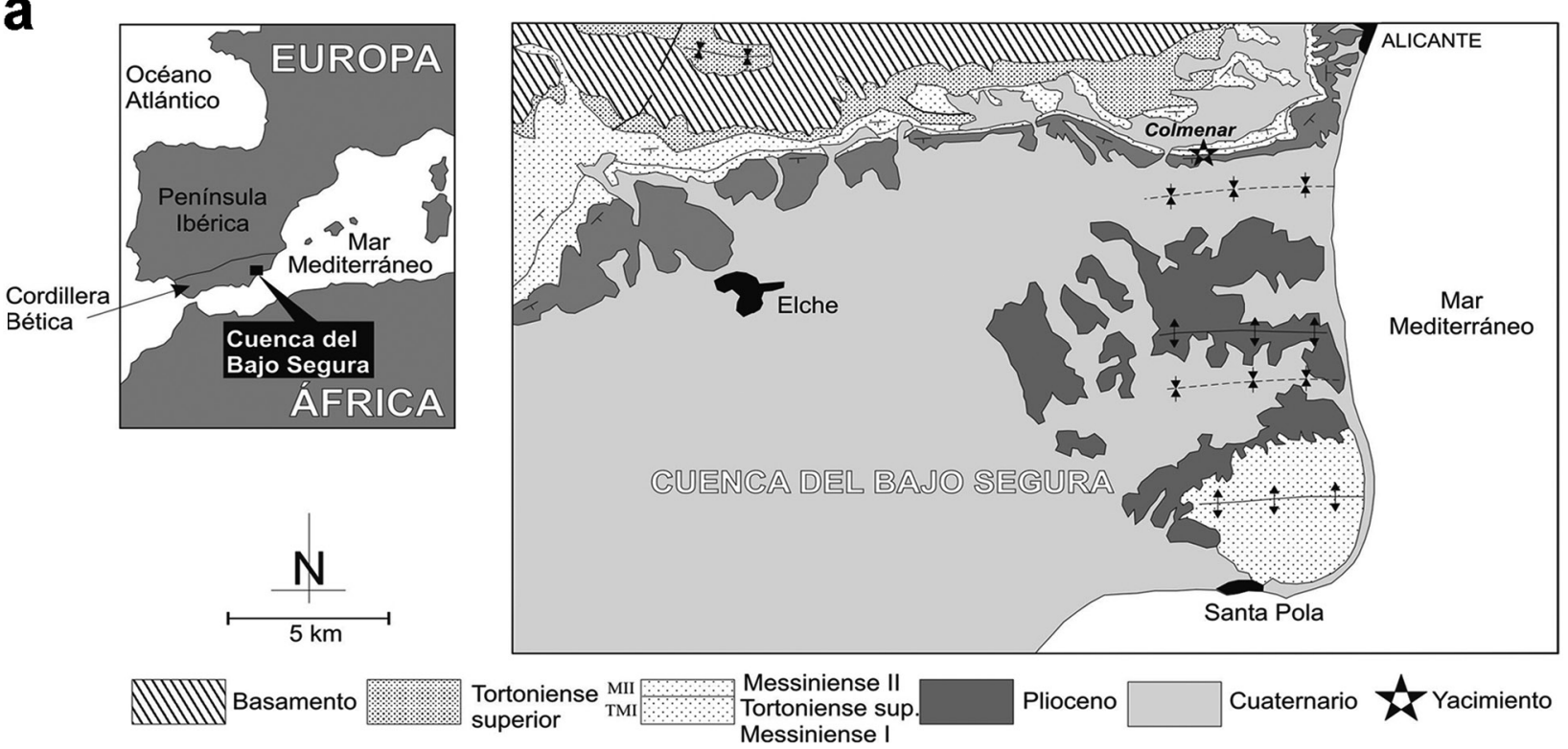

b

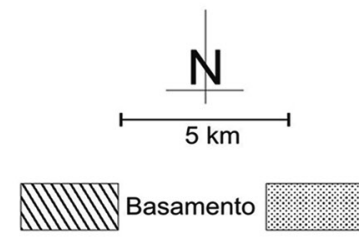
$\begin{array}{lll}\text { Tortoniense } & \text { MII } \\ \text { superior } & \text { TMI }\end{array}$ Messiniense I
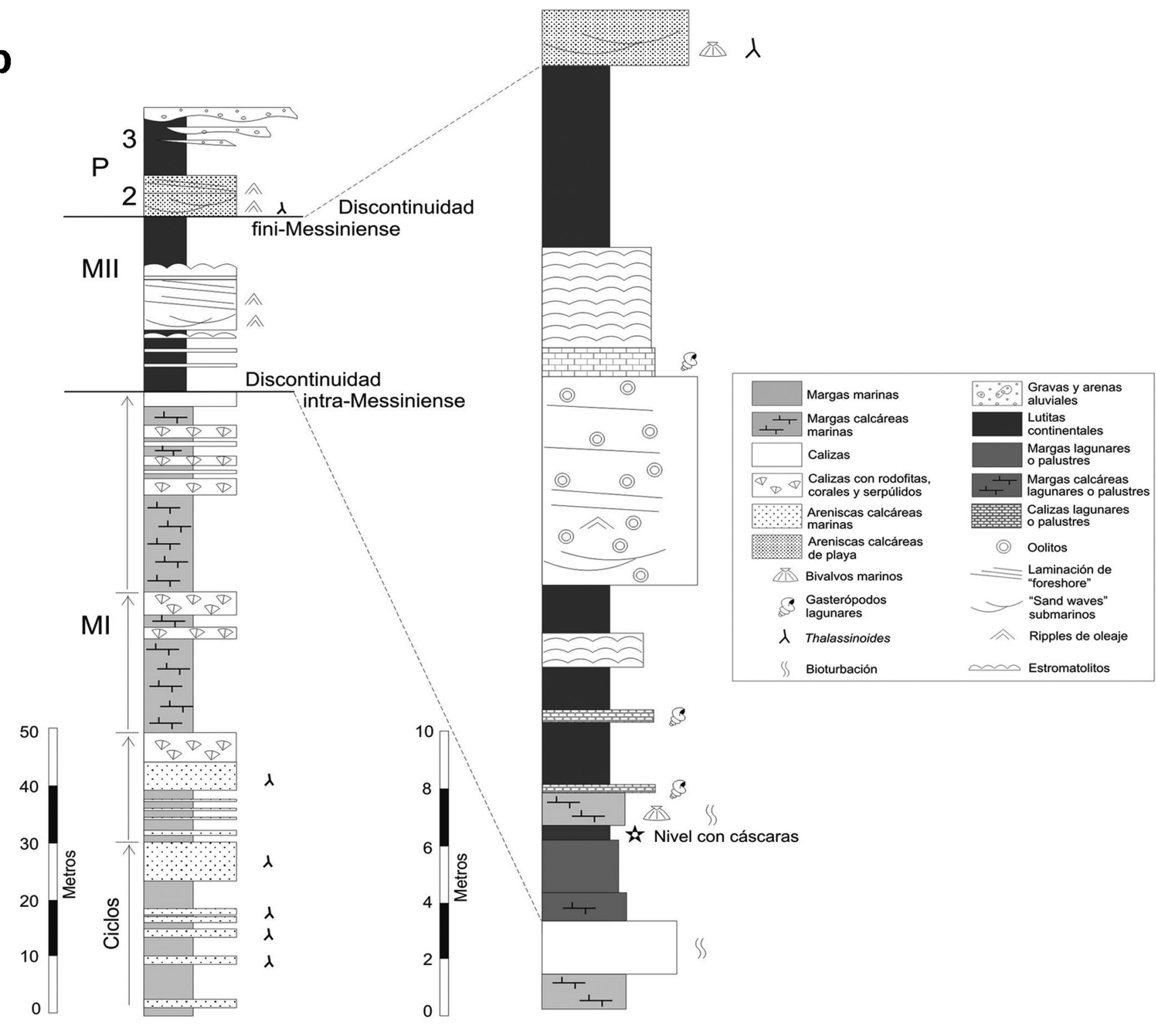
En la figura $1 \mathrm{~b}$ se ha representado la sección estratigráfica del sector de la Sierra del Colmenar en el que se encuentra el yacimiento. Se puede observar que las cáscaras aparecen en un nivel lutítico de facies continentales situado cerca de la base de la unidad aloestratigráfica MII. Dicho nivel lutítico, de $70 \mathrm{~cm}$ de espesor, está formado a su vez por dos tramos: el inferior, de lutitas rojizas, y el superior, de lutitas verdosas. Las cáscaras aparecen en la parte inferior de las lutitas verdosas y en la superior de las lutitas rojas.

En la unidad MII de sectores cercanos, como en el $\mathrm{N}$ de Elche y Crevillente, se han encontrado niveles con micromamíferos atribuidos al Turoliense superior o Messiniense, biozona MN13 (Alfaro et al., 1995; Martín Suárez \& Freudenthal, 1998). Por otra parte, la posición del yacimiento, situado bajo la discontinuidad fini-Messiniense, atestigua su edad pre-Pliocena. Así pues, se puede asegurar la edad Messiniense del yacimiento, que, con más precisión, podría asignarse al Messiniense superior.

Desde el punto de vista sedimentológico, la unidad aloestratigráfica Messiniense MII de este sector está constituida por dos sistemas deposicionales interdigitados. El primero de ellos es continental y está formado por dos conjuntos litológicos correlativos: i) calizas con gasterópodos, margas y lutitas depositadas en ambientes lagunares someros y palustres, y ii) lutitas con paleosuelos hidromórficos y pequeños canales aluviales de gravas y arenas. Correspondería por tanto a un contexto sedimentario continental constituido por lagunas litorales y facies distales de abanicos aluviales. El segundo sistema deposicional es marino litoral y está constituido por tres asociaciones de facies diferentes: i) margas calcáreas marinas con bivalvos (pectínidos y ostréidos), ii) calizas estromatolíticas, que llegan a formar cúpulas de más de un metro de altura, que se interpretan como depósitos marinos submareales de ambientes hipersalinos, y iii) calcarenitas oolíticas con frecuentes estructuras tractivas (ripples de oleaje, sand waves y laminación de foreshore) que se interpretan como depósitos de playa asociados a un sistema de isla barrera-laguna.

El yacimiento se encuentra asociado a facies del sistema deposicional continental. Se puede considerar que la puesta se realizó cerca del margen de una laguna litoral, de aguas dulces o salobres, que estaría separada del mar abierto por un cordón litoral oolítico.

\section{MATERIAL Y MÉTODOS}

Los más de 400 fragmentos de cáscara del yacimiento de Torrellano se hallaron concentrados en un área de aproximadamente $1 \mathrm{~m}^{2}$. No se han encontrado huevos completos, ni restos óseos. Todos los fragmentos se limpiaron mediante un baño de ultrasonidos y se dejaron secar a temperatura ambiente, sin utilizar productos químicos en su tratamiento. El grosor de las cáscaras se midió por medio de un micrómetro, evitando las zonas más erosionadas (con depresiones o cráteres), tomando la media de tres mediciones consecutivas como el valor final. Algunos pequeños fragmentos fueron montados en soportes de aluminio, recubiertos con oro y fotografiados mediante el microscopio electrónico de barrido (Phillips XL30). Otros fragmentos ( $\mathrm{N}=25$ ) fueron empleados para preparar láminas delgadas que se examinaron mediante el microscopio con luz normal y polarizada. Algunas cáscaras con la superficie externa bien preservada $(\mathrm{N}=148)$ fueron examinadas con una lupa binocular para la descripción de la morfología de los poros. La nomenclatura empleada sigue la utilizada por Sauer (1972) y Mikhailov (1991, 1997).

El sistema parataxonómico desarrollado en las últimas décadas para la clasificación de las cáscaras y huevos fósiles es aceptado en la actualidad por la mayoría de los autores. Este sistema aboga por el empleo de la raíz "oolithus" en la nomenclatura de las oofamilias y oogéneros (Mikhailov et al., 1996). En el caso de las dos oospecies de Namibia, los autores discrepan de la aproximación parataxonómica ya que consideran que los huevos son una parte de las especies como "lo son los huesos, plumas y tejidos blandos" (Pickford et al., 1995). De este modo, no emplean el sufijo -oolithus sino -ornis, lo cual puede llevar a cierta confusión al poder considerarse que Diamantornis wardi y Diamantornis corbetti se refieren a los restos óseos de un ave. No es objetivo del presente trabajo la revisión de la nomenclatura de dichas ooespecies, por lo que se han mantenido dichas denominaciones.

La codificación de los caracteres de las cáscaras de ratites actuales y de dinornítidos se basa en la reciente revisión de Zelenitsky \& Modesto (2003), así como en Tyler \& Simkiss (1959). En el caso de Dinornithidae (moas), estos autores consideran los caracteres de cáscaras finas y gruesas, atribuibles probablemente a varias especies de moas. Los datos sobre las dos ooespecies procedentes del Mioceno de Namibia (Diamantornis wardi Pickford, Senut \& Dauphin, 1995, y Diamantornis corbetti Pickford, Senut \& Dauphin, 1995) provienen tanto de la bibliografía (Pickford et al., 1995; Dauphin et al., 1996), como de la observación directa del material.

La finalidad del análisis cladístico es corroborar que los nuevos caracteres descritos también están relacionados con la filogenia, y por tanto son válidos como elementos de clasificación. Un segundo objetivo sería, mediante este tipo de análisis, llevar a cabo una primera aproximación filogenética para el taxón de Torrellano, así como para Diamantornis corbetti y Diamantornis wardi, conocidos únicamente a partir de fragmentos de cáscaras. Por ello, antes de incluir estos fósiles en un análisis filogenético, se ha comprobado la fiabilidad de los caracteres definidos a la hora de realizar este tipo de análisis, para lo cual se ha realizado un primer análisis incluyendo sólo los taxones actuales, cuyas relaciones filogenéticas ya han sido estudiadas. Esto permite comparar el cladograma resultante con aquellos realizados a partir de caracteres moleculares o esqueléticos. 
Tras comprobar que los caracteres definidos son filogenéticamente útiles, se determinaron los estados de carácter correspondientes para los fósiles. Posteriormente, se repitió el análisis cladístico incluyendo a los fósiles, para identificar su posición dentro del cladograma.

A la hora de realizar los análisis filogenéticos se ha utilizado como grupo externo o outgroup al género Tinamus, perteneciente a la familia Tinamidae, tradicionalmente aceptada como grupo hermano más probable de ratites, aunque un estudio reciente (Harshman et al., 2008) lo incluye dentro de ratites, implicando un origen polifilético para este último grupo. En el primero de los análisis el grupo interno o ingroup está conformado por los géneros actuales Apteryx, Dromaius, Casuarius, Struthio y Rhea. En el segundo análisis, se han añadido los fósiles: material de Torrellano, Diamantornis wardi y Diamantornis corbetti, así como a Dinornithidae.

Estos análisis filogenéticos han sido realizados mediante el algoritmo de búsqueda exhaustiva del programa PAUP* $4.0 b 10$ (Swofford, 2002). En ambos casos se utilizaron once caracteres discretos, y se evitó el empleo de caracteres continuos que, por otra parte, suelen ser utilizados en análisis referentes a cáscaras, ya que su variabilidad no está estadísticamente estudiada, y por tanto es difícil delimitar claramente los estados de carácter. Los caracteres utilizados hacen referencia a la parte inorgánica de la cáscara que es lo que mejor se preserva en el material fósil (Anexo). Por último, los once caracteres utilizados (tabla 1) fueron descritos como no ordenados (y por tanto no polarizados), ya que la secuencia evolutiva de estados de carácter de las cáscaras no se conoce actualmente.

El material se encuentra depositado temporalmente para su estudio en la Unidad de Paleontología, Departamento de Biología de la Universidad Autónoma de Madrid.

\section{RESULTADOS}

\section{DESCRIPCIÓN DEL MATERIAL}

La mayor parte de las cáscaras aparecen fuertemente erosionadas, mostrando ambas superficies irregulares y con pequeñas depresiones. Debido a esta erosión los poros han podido estudiarse sólo en una parte de la muestra (aproximadamente el 30\%). En este material bien preservado, los poros pueden aparecer aislados, de tamaño relativamente pequeño, circulares o ligeramente ovalados, o bien encontrarse en el interior de surcos alargados, bifurcados o irregulares. En vista radial, los canales respiratorios son rectos, pero algunos de ellos se bifurcan o ramifican en la zona más externa de la cáscara, de modo que dos poros abren en el interior de un mismo surco. Estos surcos pueden ser más o menos rectos, paralelos entre sí o bien aparecer varios surcos próximos perpendiculares entre sí, o radiando desde un punto (Figs. 2a, 2b y 2c).

La superficie interna está cubierta por mamilas de pequeño tamaño, empaquetadas y sin preservar los eisosferitos (agregados de cristales de calcita) de la base de las mamilas.

En vista radial, la cáscara consta de tres capas: una capa basal o capa mamilar (ML), en la que se pueden distinguir los prismas que forman las mamilas más o menos perpendiculares a la superficie de la cáscara, seguida de una capa de aspecto más uniforme o capa continua (CL) y una tercera capa muy fina, denominada zona externa (EZ). Las mamilas son más altas que anchas y están formadas por una sola capa de cristales de calcita en forma de cuña que radian desde un punto basal o núcleo orgánico (Figs. 2d, 2e). La transición entre las capas continua y mamilar es gradual, y sólo en las láminas delgadas es posible medir el tamaño de la ML, que es aproximadamente dos veces más gruesa que la CL (ratio $\mathrm{ML}: \mathrm{CL}=2: 1$ ) (Fig. 2f).

\begin{tabular}{|c|c|c|c|c|c|c|c|c|c|c|c|}
\hline & 1 & 2 & 3 & 4 & 5 & 6 & 7 & 8 & 9 & 10 & 11 \\
\hline Tinamus & 0 & 0 & 0 & 0 & 0 & 0 & 0 & 0 & 0 & 0 & 0 \\
\hline Struthio & 1 & 0 & 1 & 1 & 1 & 1 & 1 & 1 & 2 & 1 & 0 \\
\hline Rhea & 1 & 0 & 0 & 1 & 1 & 1 & 1 & 1 & 1 & 0 & 0 \\
\hline Casuarius & 1 & 1 & 0 & 1 & 1 & 1 & 1 & 1 & 1 & 3 & 1 \\
\hline Dromaius & 1 & 1 & 0 & 1 & 1 & 1 & 1 & 1 & 1 & 3 & 1 \\
\hline Apteryx & 0 & 0 & 0 & 0 & 0 & 0 & 0 & 1 & 0 & 0 & 0 \\
\hline Dinornithidae & 0 & 0 & 0 & 1 & 1 & 0 & 0 & 1 & 1 & 1 & 0 \\
\hline Torrellano & 1 & 0 & 1 & 1 & 1 & 0 & 0 & 1 & 1 & 2 & 0 \\
\hline Diamantornis wardi & 2 & 0 & 1 & 1 & 1 & 0 & $\ldots$ & 1 & 2 & 1 & 0 \\
\hline Diamantornis laini & 1 & 0 & 1 & 1 & 1 & 0 & $?$ & 1 & 2 & 1 & 0 \\
\hline
\end{tabular}

Tabla 1. Matriz de datos de la distribución de estados de carácter para los once caracteres de las cáscaras de ratites utilizados. Data matrix of character-state distribution for the eleven eggshell characters of ratites used. 

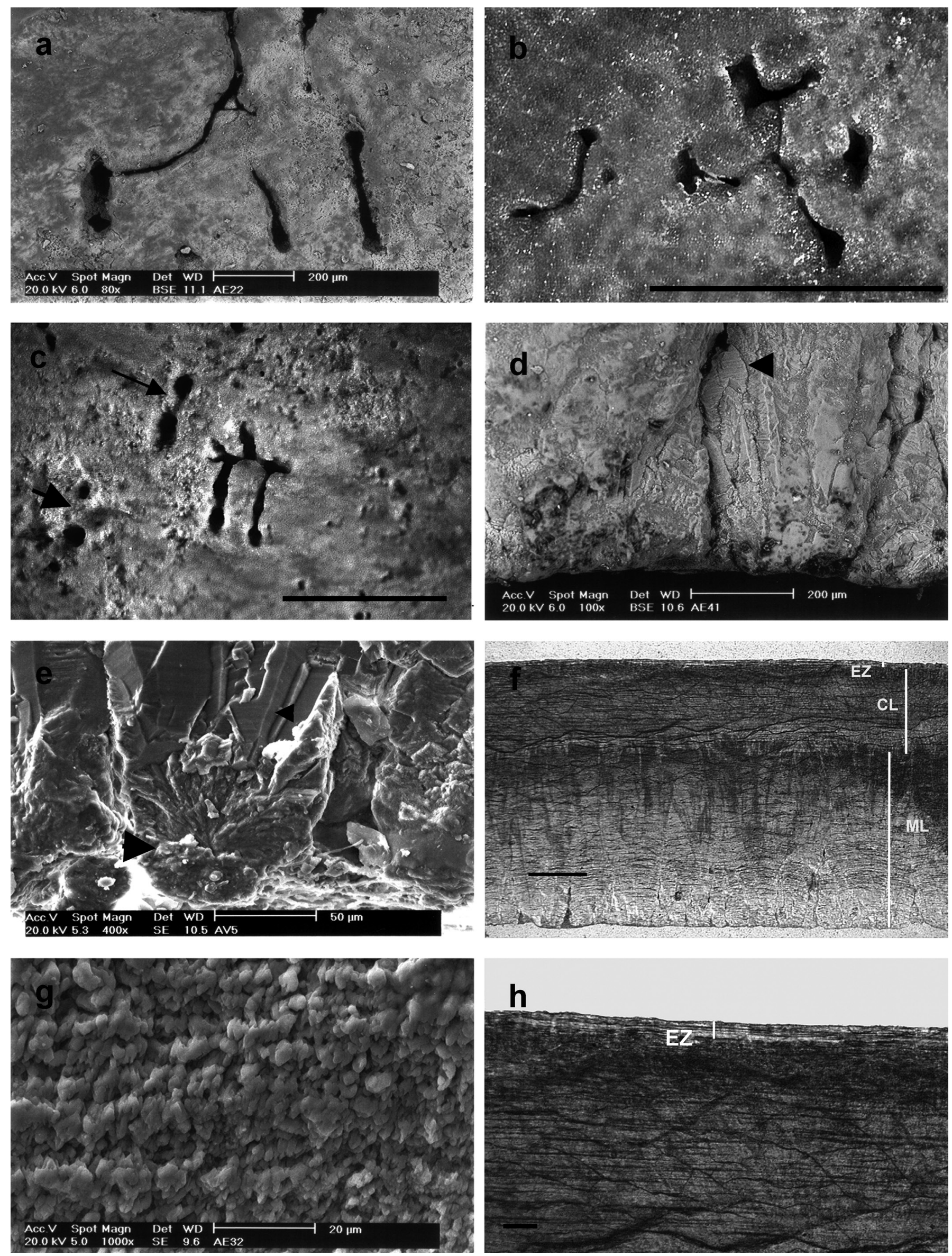

\section{h}

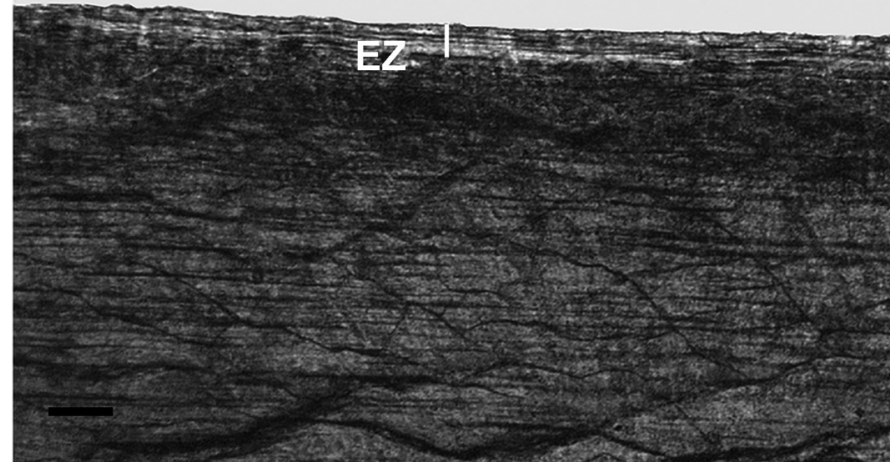


En la capa continua los cristales de calcita tienen forma de escamas irregulares, lo que se conoce como ultraestructura escamosa (Mikhailov, 1997) (Fig. 2g). Las líneas de crecimiento aparecen desde la base a la superficie de la cáscara, ligeramente curvadas en el primer tercio de la capa mamilar y pasando gradualmente a ser horizontales hasta llegar a la superficie. No se han observado vesículas ni en la capa mamilar ni en la continua. El grosor de la cáscara varía entre 2,1-3,0 mm (media de 2,6 mm), aunque estos valores podrían estar alterados por la erosión, a pesar de haberse evitado en la medición las zonas más alteradas de las cáscaras. Finalmente, a continuación de la CL aparece la zona externa (EZ), que consiste en una fina capa (aproximadamente 2\% del grosor total) formada por cristales de calcita verticales, que sólo puede ser observada en algunas cáscaras bien preservadas (Fig. 2h).

\section{ANÁLISIS CLADÍSTICO}

En este análisis se han empleado 11 caracteres microy ultraestructurales de cáscaras de ratites actuales y fósiles (ver anexo). La codificación de dichos caracteres se encuentra en la tabla 1 .
El primero de los análisis llevados a cabo, incluyendo sólo taxones actuales ha generado tres árboles de máxima parsimonia (Longitud=13; CI=1,000 excluyendo caracteres no informativos, números 3 y 8 en este caso; RI=1,000; $\mathrm{RC}=0,714)$.

El cladograma consenso estricto obtenido muestra a Apteryx como la ratite más basal y a los géneros Casuarius y Dromaius formando un clado, hermanado mediante una politomía con los géneros Struthio y Rhea (Fig. 3a).

El segundo de los análisis realizados, incluyendo esta vez el material fósil, ha generado 16 árboles de máxima parsimonia (Longitud=18; $\mathrm{CI}=0,824$ excluyendo caracteres no informativos, número 8 en este caso; RI=0,857; $\mathrm{RC}=0,714)$.

El consenso obtenido a partir de estos 16 árboles por el método del $50 \%$ majority rule muestra de nuevo a $\mathrm{Ap}$ teryx como ratite más basal, seguido de Dinornithidae, y por último una politomía formada por tres grupos: por un lado Torrellano aislado, por otro lado un clado formado por Casuarius y Dromaius como grupo hermano de Rhea, y como tercer grupo una nueva politomía conformada por Struthio, D. wardi y D. corbetti (Fig. 3b).

Figura 2. Fotos de material de Torrellano y de avestruz tomadas con microscopio electrónico de barrido (MEB) y lupa binocular. a, Foto al MEB de la superficie externa; los poros se encuentran en el interior de surcos alargados; nótese la irregularidad de la superficie causada por la erosión. b, Foto a la lupa binocular de la superficie externa en la que se pueden ver dos tipos de poros; a la izquierda dos poros dentro de un surco alargado y a la derecha una estructura irregular formada por varios surcos orientados radialmente (escala $1 \mathrm{~mm}$ ). c, Foto a la lupa binocular de la superficie externa (escala $1 \mathrm{~mm}$ ); a veces dos poros aparecen próximos sin estar en el interior de un surco (flecha grande); por encima aparecen otros dos poros aparentemente unidos por un fino surco (flecha pequeña); en el centro de la imagen se ven dos surcos paralelos unidos por un tercero perpendicular a ellos. d, Foto al MEB, detalle de la zona basal de las mamilas; a pesar de la erosión es posible observar que las mamilas están formadas por una sola capa de cristales de calcita en forma de cuña con ultraestructura tabular (flecha). e, Foto al MEB de detalle de la zona mamilar de una cáscara de avestruz (Struthio); se puede ver una zona basal formada por pequeños cristales radiantes (flecha grande), que se continúa en una capa formada por cristales más grandes con ultraestructura tabular (flecha pequeña). f, Foto a la lupa binocular de una lámina delgada de una cáscara del yacimiento de Torrellano; se pueden observar las tres capas horizontales que forman la cáscara (ML, CL y EZ) y su transición gradual entre ellas (escala $1 \mathrm{~mm}$ ). g, Foto al MEB de detalle de la capa continua, en la que se observa su ultraestructura escamosa. h, Foto a la lupa binocular de una lámina delgada; detalle de la zona más externa de la cáscara en la que se puede observar la EZ (escala $50 \mu \mathrm{m}$ ). El material se encuentra depositado temporalmente para su estudio en la Unidad de Paleontología, Departamento de Biología de la Universidad Autónoma de Madrid.

Photographs of Torrellano eggshells under scanning electron microscope (SEM) and stereoscopic microscope. a, SEM photograph of the outer surface; the pores open inside elongated grooves; notice the irregularity of the surface caused by erosion. $\boldsymbol{b}$, Photograph under stereoscopic microscope of the outer surface; two types of pores can be observed: left, two pores inside an elongated groove; right, irregular structure formed by some radiating grooves (scale bar $1 \mathrm{~mm}$ ). $\mathrm{c}$, Photograph under stereoscopic microscope of the outer surface; sometimes two pores open near to each other, but not inside a groove (big arrow); above, two other pores appear connected by a thin groove (small arrow); in the middle, two parallel grooves are connected by a third perpendicular groove (scale bar $1 \mathrm{~mm}$ ). d, SEM photograph, detail of the basal mamillary zone; despite of strong erosion it is possible to observe that mamillae are composed of a single layer of calcite wedges, with tabular ultrastructure (arrow). e, SEM photograph of the mamillary zone of an ostrich eggshell (Struthio); the mamillae are composed of a basal zone of small radiant crystals (big arrow), and a zone with crystal wedges of tabular ultrastructure (small arrow).f, Photograph under stereoscopic microscope of a thin slide; the eggshell show three horizontal layers (ML, CL, and EZ), with a gradual transition between them (scale bar $1 \mathrm{~mm}$ ). $\mathrm{g}$, SEM photograph of detail of the CL with a squamatic ultrastructure. $f$, Photograph under stereoscopic microscope of a thin slide of an eggshell from Torrellano site; detail of the outermost part of the eggshell, where the external zone, EZ can be observed (scale bar 50 $\mu \mathrm{m}$ ). Eggshell material deposited temporally at Unidad de Paleontología, Departamento Biología, Universidad Autónoma de Madrid. 


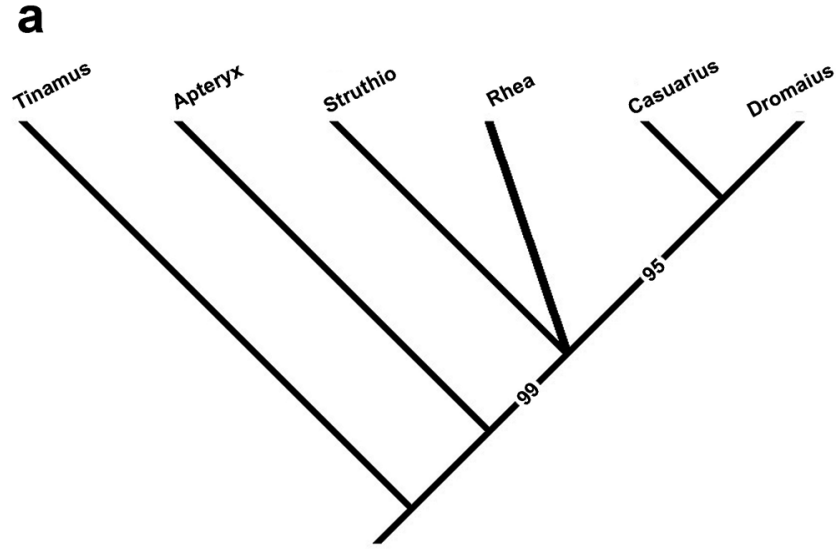

b

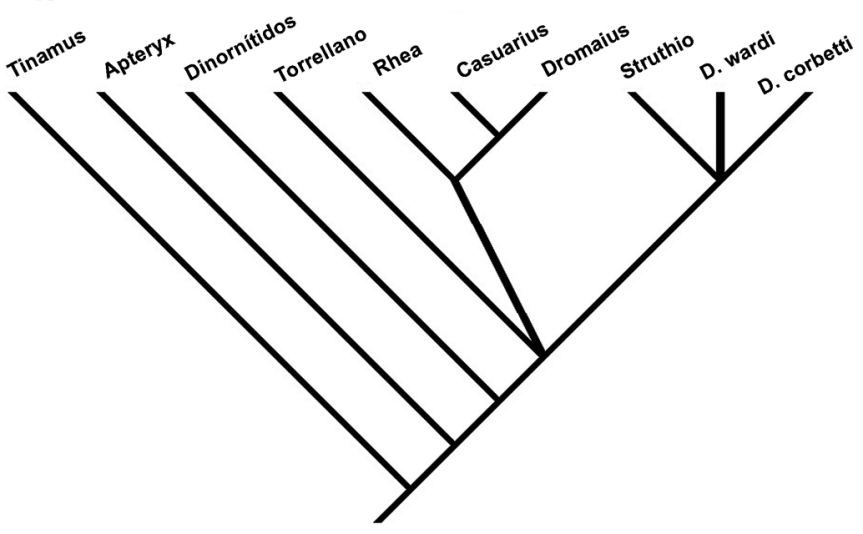

Figura 3. a, Cladograma consenso estricto obtenido a partir de los tres árboles más parsimoniosos resultantes del análisis de los caracteres de taxones actuales. b, Cladograma consenso obtenido por el método del $50 \%$ majority rule a partir de los 16 árboles más parsimoniosos resultantes del análisis conjunto de taxones actuales y material fósil.

a, Strict consensus cladogram of three most parsimonious trees resulting from the analysis of extant taxa characters. b, Consensus cladogram (50\% majority rule) of 16 most parsimonious trees resulting from the combined analysis of extant taxa and fossil material characters.

\section{DISCUSIÓN}

\section{CARACTERES DE LAS CÁSCARAS}

Hasta el momento en la Península Ibérica sólo se conocía un yacimiento con cáscaras de tipo Aepyornis en el Plioceno basal de la Cuenca de Teruel (Mein \& Dauphin, 1995), lo que hace del material de Torrellano el más antiguo de este tipo para esta región. El registro de cáscaras similares se extiende por varios continentes dentro de un rango temporal que abarca desde el Eoceno hasta el Plioceno (Sauer, 1972). A pesar de este relativo elevado número de hallazgos, las descripciones se basan fundamentalmen- te en el grosor y la forma de los poros. Aunque, como ya se ha dicho, el material de Torrellano aparece erosionado, las medidas de grosor hechas evitando las zonas más alteradas, parecen suficientes para establecer comparaciones generales con material similar de otros yacimientos. Así, si comparamos los datos de grosor del registro de cáscaras de tipo Aepyornis o del género Aepyornis de distintas localidades y edades publicados por diversos autores (Tabla 2) se puede ver que el material de Torrellano presenta un rango de grosor similar al de Turquía y Lanzarote y en menor medida con el de Marruecos y La India, ya que difieren bastante en sus valores mínimos. En cuanto a las cáscaras de Teruel (Mein \& Dauphin, 1995) proporcionan el valor medio de grosor $(3,1 \mathrm{~mm})$, que es aproximado al valor obtenido en Torrellano (2,6 mm). Cabe destacar que el grosor de las cáscaras de tipo Aepyornis halladas en Namibia es el menor conocido, siendo prácticamente la mitad de gruesas que las del género Aepyornis (ver Sauer, 1972). Estas últimas alcanzan los valores máximos, sobrepasando en algunos casos los cuatro milímetros de grosor.

El grosor de una cáscara es un carácter que se encuentra influido por diversos factores biológicos como el tamaño de la hembra, grupo taxonómico, disponibilidad de ciertos nutrientes, etc, y factores externos como la presencia de sustancias tóxicas. En el caso del material fósil hay que tener en cuenta además los factores tafonómicos (transporte, erosión, reemplazamiento, etc), que también pueden alterar en mayor o menor medida este carácter. Por tanto, aunque el grosor nos puede proporcionar una primera aproximación a la identificación de una cáscara (por ejemplo, el caso de una ratite de gran tamaño), éste resulta ser un carácter sólo relativamente informativo.

Por otro lado, el carácter empleado fundamentalmente para distinguir las cáscaras de tipo Aepyornis de las de otras aves semejantes es el de la forma de los poros. Sauer (1972) en su revisión de las cáscaras fósiles procedentes de Asia, África y Europa describe tres morfologías distintas de poros, siendo la más común los surcos alargados, paralelos entre sí y al eje mayor del huevo, en cuyo interior aparecen normalmente dos poros. Este patrón ha sido observado en todo el material identificado como "aepyornitoide" o de tipo Aepyornis. En las cáscaras de Torrellano aparecen algunos poros individuales, aunque lo más común es la presencia de dos poros en el interior de un surco alargado, o bien dos poros alineados sin estar dentro de un surco. Existen además surcos orientados en distintas direcciones, y que abren próximos entre sí formando estructuras más complejas. Sauer (1972) cita también la presencia de poros que adoptan patrones difusos o en remolino. En cualquier caso, este tipo de poros refleja la estructura del sistema de canales respiratorios, el cual está formado por tubos simples y rectos, o bien bifurcados que dan lugar a dos aberturas (poros) que abren en un mismo plano, a diferencia de lo que sucede con los canales ramificados de Struthio (ver Tyler \& Simkiss, 1959). 
LOCALIDAD

\begin{tabular}{lllll}
\hline $\mathbf{1}$ & Mongolia & Eoceno superior & 2,2 & Sauer, 1972 \\
\hline $\mathbf{2}$ & Namibia & Mioceno & $1,20-1,55$ & Senut et al., 1995 \\
\hline $\mathbf{3}$ & Torrellano (Elche) & Mioceno & $2,13-3,05$ & En este trabajo \\
\hline $\mathbf{4}$ & Turquía & Mioceno-Plioceno & $1,85-3,05$ & Mein \& Dauphin, 1995 \\
\hline $\mathbf{5}$ & Marruecos & Mioceno-Plioceno & $1,40-2,65$ & Mein \& Dauphin, 1995 \\
\hline $\mathbf{6}$ & Lanzarote (Islas Canarias) & Mioceno-Plioceno & $2,10-2,90$ & Sauer \& Rothe, 1972 \\
\hline $\mathbf{7}$ & Punjab (La India) & Plioceno inferior? & $2,50-2,90$ & Sauer, 1972 \\
\hline $\mathbf{8}$ & Teruel (España) & Plioceno & 3,1 & Mein \& Dauphin, 1995 \\
\hline $\mathbf{9}$ & St. Augustine (Madagascar) & $? ?$ & 3,3 & Sauer, 1972 \\
\hline $\mathbf{1 0}$ & Ampamalora (Madagascar) & $1970+/-90$ (años/years) & $2,88-3,55$ & Sauer, 1972 \\
\hline $\mathbf{1 1}$ & Tulear (Madagascar) & $5210+/-140$ (años/years) & $3,13-4,30$ & Sauer, 1972 \\
\hline
\end{tabular}

Tabla 2. Datos bibliográficos de rangos o valores medios de grosor de cáscaras del género Aepyornis o de tipo Aepyornis, procedentes de diversas localidades y edades.

Bibliographic data of thickness ranges or means of genus Aepyornis or Aepyornis type eggshells from different localities and ages.

En cuanto a la microestructura, son muy escasas las descripciones en la literatura. Mein \& Dauphin (1995) citan la existencia de tres capas microestructurales en el material de Teruel, mientras que Senut et al. (1995), sólo mencionan la presencia de dos capas en el material de Namibia. Las cáscaras de Torrellano presentan también tres capas con distinta ultraestructura, siendo la capa más interna (ML) el doble de gruesa que la capa continua. En corte fresco bajo la lupa binocular, o incluso con el MEB es difícil establecer los límites entre ambas capas, ya que sólo es claramente visible la zona más basal de las mamilas. Esto podría explicar las diferencias entre el índice ML:CL del material de Torrellano, y el de cáscaras similares halladas en Namibia (ML:CL=0,25-0,40; Dauphin et al., 1996), aunque este último material, como ya se ha visto, también presenta un grosor mucho menor. En cualquier caso, el carácter "índice ML:CL" podría ser una sinapomorfía de las cáscaras de tipo Aepyornis o incluso del propio género Aepyornis, sin embargo la ausencia de este dato en la bibliografía no permite por el momento comprobar esta hipótesis, quedando pendiente para su desarrollo en futuros trabajos.

\section{ANÁLISIS CLADÍSTICO}

El árbol consenso derivado del primer análisis, en el que únicamente se incluyeron taxones actuales, es completamente congruente con el obtenido por Zelenitsky \& Modesto (2003) llevado a cabo también analizando únicamente caracteres de cáscaras. En él se sugiere una posición basal para Apteryx dentro de Ratitae. Un análisis de Bremer de este conjunto de datos indica que el nodo que hermana a Apteryx con el clado formado por Rhea, Struthio, Casuarius y Dromaius es muy robusto, puesto que serían necesarios cinco pasos adicionales para colapsar dicho nodo (es decir, que el árbol más parsimonioso en el que no apareciesen estos dos clados como grupos hermanos tendría una longitud de dieciocho pasos, cinco más que el árbol consenso estricto obtenido). El clado formado por Casuarius y Dromaius también es relativamente robusto, ya que un análisis de Bremer indica que para colapsar el nodo que los une serían necesarios dos pasos adicionales. El clado que agrupa a Rhea, a Struthio y al clado formado por Casuarius y Dromaius, por el contrario, no puede ser resuelto mediante datos procedentes de las cáscaras, tal y como ocurre en el análisis de Zelenitsky \& Modesto (2003), y permanece por tanto como una politomía.

Si se compara con otras hipótesis filogenéticas logradas a partir de datos esqueléticos, como la de Lee et al. (1997), se puede ver que el árbol obtenido es consistente con éstas, ya que una de las posibilidades incluidas en la politomía del presente análisis coincide con la resolución obtenida en el anteriormente citado. El nodo que hermana a Apteryx con el resto de ratites actuales, así como el clado formado por Casuarius y Dromaius, no sólo son consistentes con el árbol logrado a partir de datos esqueléticos, sino que son congruentes con él.

Comparando con hipótesis filogenéticas obtenidas a partir de datos moleculares (Lee et al., 1997; Haddrath \& Bakker, 2001; Cooper et al., 2001) se observa que una vez más, el clado formado por Casuarius y Dromaius se mantiene, y por tanto su posición es congruente con la resolución obtenida en nuestro análisis. Por el contrario, en todas estas hipótesis también aparece de manera constante la situación de Apteryx como grupo hermano de este clado, de manera que las posiciones relativas del resto no son equivalentes en análisis realizados con datos moleculares y con datos morfológicos. 
El hecho de que nuestros resultados concuerden con los obtenidos a partir de análisis realizados con datos morfológicos, y en parte también con los obtenidos a partir de datos moleculares, sugieren que los caracteres de las cáscaras escogidos están relacionados con la filogenia y, por tanto, es razonable utilizarlos como criterios de clasificación filogenética. Además, esta afirmación se ve reforzada por el hecho de que el índice de consistencia obtenido para los árboles filogenéticos es de 1, lo cual implica que ninguno de los caracteres presenta homoplasia. Teniendo esto en cuenta se realizó el segundo análisis filogenético, en el que se incluyeron los grupos fósiles.

El árbol consenso resultante de este segundo análisis vuelve a mostrar a Apteryx como la ratite más basal, formando un nodo en el que se hermana con el resto de ratites, algo congruente con los resultados de análisis morfológicos, ya sea teniendo en cuenta sólo caracteres esqueléticos (Lee et al., 1997) o también de las cáscaras (Zelenitsky $\&$ Modesto, 2003). En un análisis de remuestreo aleatorio bootstrap con cinco mil réplicas se obtiene un porcentaje del $99 \%$ para la aparición de este nodo que separa a Apteryx del resto de ratites. El siguiente taxón más basal es Dinornithidae, que ocupa una posición basal en todos los análisis llevados a cabo (Lee et al., 1997; Haddrath \& Bakker, 2001; Cooper et al., 2001; Zelenitsky \& Modesto, 2003) y, en este caso, la solución es congruente con otros análisis de datos morfológicos. El siguiente nodo muestra una politomía formada por tres clados: el primero lo forma Torrellano, el segundo está constituido por Rhea, Casuarius y Dromaius, y el tercero por Struthio, D. corbetti y D. wardi.

Las cáscaras de tipo Aepyornis del yacimiento de Torrellano aparecen en una posición similar a la que ocupa Aepyornis en los análisis moleculares (Haddrath \& Bakker, 2001; Cooper et al., 2001), es decir, se mantienen las mismas relaciones filogenéticas excepto para Apteryx y Rhea que intercambian sus posiciones (en nuestro análisis $A p$ teryx ocupa una posición basal, mientras que en los análisis moleculares esta posición está ocupada por Rhea). Así, se aprecia cómo el material de Torrellano aparece asociado a Struthio, Casuarius y Dromaius y, en cualquier caso, formando parte del crown group de ratites, y no en una posición basal como Apteryx o Dinornithidae.

En el segundo clado de la politomía, Casuarius y Dromaius vuelven a aparecer como grupos hermanos; el análisis de remuestreo de cinco mil réplicas concede un $95 \%$ de soporte para el nodo que los une. La posición de Rhea como grupo hermano de este clado es consistente con los análisis realizados a partir de caracteres morfológicos, pero no con caracteres moleculares, ya que en estos Rhea suele ocupar una posición basal dentro de Ratitae. La posición de Struthio, incluido en el tercer clado de la politomía, respecto a la de Rhea, Casuarius y Dromaius es incongruente pero, en general, no inconsistente con los análisis tanto morfológicos como moleculares. Es decir, si bien la resolución del cladograma no es idéntica a las de estos estudios, nuestra politomía si incluye sus resoluciones. Resumiendo, Struthio generalmente ocupa una posición muy próxima a Rhea, Casuarius y Dromaius.

$D$. corbetti y D. wardi aparecen unidos a Struthio mediante una politomía que los caracteres de las cáscaras no logran resolver. Conviene recordar que $D$. corbetti y $D$. wardi son parataxones $\mathrm{y}$, por lo tanto, no tienen por qué guardar una relación más estrecha entre ellos que con Struthio. Por otra parte, a pesar de que $D$. corbetti y $D$. wardi no han sido incluidos anteriormente en ningún análisis cladístico junto con otras ratites, el parataxón Diamantornis sí ha sido asignado a la familia Struthionidae aunque de manera no definitiva (Bibi et al., 2006).

En comparación con los resultados de Zelenitsky \& Modesto (2003), el árbol obtenido es congruente con el realizado por éstos a partir únicamente de caracteres de cáscaras, ya que la resolución en el presente trabajo de la politomía que ellos representan para el crown group de ratites está incluida en ésta. Respecto a su hipótesis filogenética obtenida a partir de datos esqueléticos y de las cáscaras, el árbol presentado en este trabajo también es congruente, excepto en la posición relativa de Rhea y Struthio. Teniendo esto en cuenta, y a pesar de haber utilizado diferentes caracteres, se puede concluir una vez más que, efectivamente, las cáscaras presentan una señal filogenética constante, es decir, cuya información no varía dependiendo de qué caracteres se elijan, y que es, además, congruente con la información derivada de los datos esqueléticos, y en menor medida, también moleculares.

Un estudio reciente (Harshman et al., 2008) presenta un análisis cladístico a partir de secuencias de ADN nuclear. En contraposición a los estudios anteriores, este trabajo defiende un origen polifilético para ratites, ya que Tinamidae quedaría incluido dentro de estas. A partir de este resultado interpretan que es más plausible una evolución convergente de los caracteres morfológicos asociado a la pérdida de la capacidad de vuelo en ratites, que el hecho de que estos estén relacionados con la filogenia. Sin embargo, la arquitectura del cladograma obtenido por estos autores, no varía drásticamente respecto a las anteriores, y por lo tanto, sí parece lógico pensar que exista dicha relación entre los caracteres morfológicos y la filogenia. Del mismo modo, los resultados obtenidos en el presente estudio sugieren que los caracteres de las cáscaras reflejan más una asociación con la filogenia que una evolución convergente. Sería, por tanto, interesante profundizar en este tema en trabajos futuros, incluyendo taxones actuales que no limiten tanto la elección de caracteres.

\section{AGRADECIMIENTOS}

El acceso al material fósil de las oospecies Diamantornis wardi y Diamantornis corbetti ha sido posible gracias a la co- 
laboración de los Drs. Jorge Morales y Martin Pickford. Nuestro agradecimiento a la Dra. Nieves López-Martínez y al Dr. Joaquín Moratalla por sus comentarios y sugerencias que han contribuido a la sustancial mejora del presente trabajo. Este estudio ha sido financiado en parte por una beca Jurassic Foundation concedida a uno de los autores (A. M. Bravo).

\section{BIBLIOGRAFÍA}

Alfaro, P., Soria, J.M. \& Ruiz Bustos, A. 1995. Precisiones biostratigráficas y paleoecológicas en el Neógeno de la Cuenca del Bajo Segura (Cordillera Bética Oriental). Estudios Geológicos, 51, 57-63.

Balanoff, A. 2003. Aepyornis maximus (On-line). Digital Morphology. http://digimorph.org/specimens/Aepyornis_ maximus/

Bibi, F., Shabel, A., Kraatz, B. \& Stidham, T. 2006. Fossil Ratite (Aves: Palaeognathae) eggshell discoveries from the Late Miocene Baynunah Formation of the United Arab Emirates, Arabian Peninsula. Palaeontologia Electronica, 9, Issue 1, 2A 13pp.

Cooper, A., Lalueza-Fox, C., Anderson, S., Rambaut, A., Austin, J. \& Ward, R. 2001. Complete mitochondrial genome sequences of two extinct moas clarify ratite evolution. Nature, 409, 704-707.

Dauphin, Y., Pickford, M. \& Senut, B. 1996. Microstructures des coquilles d'oeufs d'oiseaux fossiles de Namibie. Revue de Paléobiologie, 15, 225-241.

Fuller, E. 2001. Extinct Birds. Oxford University Press, 400 pp.

Garcia, G. \& Vianey-Liaud, M. 2001. Dinosaur eggshells as biochronological markers in Upper Cretaceous continental deposits. Palaeogeography, Palaeoclimatology, Palaeoecology, 169, 153-164.

Garcia, G., Marivaux, L., Pélissié, T. \& Vianey-Liaud, M. 2006. Earliest Laurasian sauropod eggshells. Acta Paleontologica Polonica, 51, 99-104.

Grellet-Tinner, G. 2000. Phylogenetic interpretation of eggs and eggshells of Paleognathae. In: First International Symposium on Dinosaur Eggs and Babies (Eds. A.M. Bravo \& T. Reyes). Isona, Extended Abstracts, 61-75.

Haddrath, O. \& Bakker, A.J. 2001. Complete mitochondrial DNA genome sequences of extinct birds: ratite phylogenetics and the vicariance biogeography hypothesis. Proceedings of the Royal Society of London, B, Biological Sciences, 268, 939-945.

Harshman, J., Braun, E.L., Braun, M.J., Huddleston, C.J, Bowie, R.C.K, Chojnowski, J.L., Hackett, S.J., Han, K.L., Kimball, R.T., Marks, B.D., Miglia, K.J., Moore, W.S., Reddy, S., Sheldon, F.H., Steadman, D.W., Steppan, S.J., Witt, C.C. \& Yuri, T. 2008. Phylogenomic evidence for multiple losses of flight in ratite birds. Proceedings of the National Academy of Sciences, 105, 13462-13467.

Hirsch, K.F., Kihm A.J. \& Zelenitsky, D.K. 1997. New eggshell of ratite morphotype with predation marks from the Eocene of Colorado. Journal of Vertebrate Paleontology, 17, 360-369.
Horner, J. R. 2000. Dinosaur reproduction and parenting. Annual Review Earth Planetary Sciences, 28, 19-45.

Kohring, R. 1999. Strukturen, Biostratinomie, systematische und phylogenetische Relevanz von Eischalen amnioter Wirbeltiere. Courier Forschungsinstitut Senckenberg, 210, 1-307.

Lee, K., Feinstein, J. \& Cracraft, J. 1997. The phylogeny of ratite birds: resolving conflicts between molecular and morphological data sets. In: Avian molecular evolution and systematics (Ed. D. P. Mindell). Academic Press, San Diego, 173-211.

Martín-Suárez, E. \& Freudenthal, M. 1998. Biostratigraphy of the continental Upper Miocene of Crevillente (Alicante, SE Spain). Geobios, 31, 839-847.

Mein, P. \& Dauphin, Y. 1995. Des coquilles d'oeufs de type Aepyornis dans le bassin de Teruel (Pliocène basal, Espagne). Neues Jahrbuch Geologie und Paläontologie, Monatshefte, 1995, 182-191.

Mikhailov, K.E. 1991. Classification of fossil eggshells of amniotic vertebrates. Acta Palaeontologica Polonica, 36, 193-238.

Mikhailov, K.E. 1997. Fossil and recent eggshells in amniotic vertebrates: fine structure, comparative morphology and classification. Special Papers in Paleontology, 56, 80 pp.

Mikhailov, K.E., Bray, E. \& Hirsch, K.F. 1996. Parataxonomy of fossil egg remains (Veterovata): principles and applications. Journal of Vertebrate Paleontology, 16, 763-769.

Pickford, M., Senut, B. \& Dauphin, Y. 1995. Biostratigraphy of the Tsondab sandstone (Namibia) based on gigantic avian eggshells. Geobios, 28, 85-98.

Sauer, F. 1972. Ratite eggshells and phylogenetic questions. Bonner Zoologische Beiträge, 23, 3-48.

Sauer, F. \& Rothe, P. 1972. Ratite eggshells from Lanzarote, Canary islands. Science, 176, 43-45.

Segalen, L., Renard, M., Pickford, M., Senut, B., Cojan, I., Le Callonec, L. \& Rognon, P. 2002. Environmental and climatic evolution of the Namib Desert since the Middle Eocene: the contribution of carbon isotope ratios in ratite eggshells. Compte Rendu Geoscience, 334, 917-924.

Senut, B., Pickford, M. \& Dauphin, Y. 1995. Découverte d'oeufs de type Aepyornithoïde dans le Miocène inférieur de Namibie. Comptes Rendus de l'Academie des Sciences Paris, Ser. 2a, 320, 71-76.

Soria, J.M., Yébenes, A. \& Caracuel, J.E. 2003. Estratigrafía del Messiniense y Plioceno en el margen norte de la Cuenca del Bajo Segura (Cordillera Bética oriental). Cambios paleogeográficos asociados a la crisis de salinidad del Mediterráneo. Geotemas, 5, 219-223.

Soria, J.M., Caracuel, J.M., Yébenes, A., Fernández, J. \& Viseras, C. 2005. The strathigraphic record of the Messinian salinity crisis in the northern margin of the Bajo Segura Basin (SE Spain). Sedimentary Geology, 179, 225-247.

Swofford, D.L. 2002. PAUP*: Phylogenetic Analysis Using Parsimony (*and other methods). Version 4.0b10. Sinauer Associates, Sunderland, Mass.

Stern, L.A., Johnson, G.D. \& Chamberlain, C.P. 1994. Carbon isotope signature of environmental change found in fossil ratite eggshells from a South Asian Neogene sequence. Geology, 22, 419-422. 
Stidham, T.A. 2004. Extinct ostrich eggshell (Aves: Struthionidae) from the Pliocene Chiwondo Beds, Malawi: implications for the potential biostratigraphic correlation of African Neogene deposits. Journal of Human Evolution, 46, 489-496.

Tyler, C. \& Simkiss, K. 1959. A study of the eggshells of Ratite birds. Proceedings of the Zoological Society of London, 133, 201-243
Zelenitsky, D.K. \& Modesto, S.P. 2003. New information on the eggshell or Ratites (Aves) and its phylogenetic implications. Canadian Journal of Zoology, 81, 962-970.

Manuscrito recibido: 25 de Junio, 2008 Manuscrito aceptado: 6 de Abril, 2009

\section{ANEXO. DESCRIPCIÓN DE CARACTERES}

1. Zona externa: gruesa (0), fina (1), ausente (2).

2. Resistant layer: presente (1), ausente (0). Esta capa está presente en los géneros actuales Casuarius y Dromaius, y se considera como una extensión modificada de la capa continua (Zelenitsky \& Modesto, 2003) en lugar de considerarlo como un homólogo de la zona externa (Grellet-Tinner, 2000).

3. Transición ML-CL: gradual (1), abrupta (0). En la reciente revisión de los caracteres de las cáscaras de ratites se señala que el género Struthio es único ya que la transición entre capas CL y ML es gradual y no abrupta como ocurre con otras ratites (Zelenitsky \& Modesto, 2003).

4. Vesículas CL: presente (0), ausente (1).

5. Vesículas ML: presente (0), ausente (1).

6. Estructura ML: una capa (0), dos capas (1).

7. Ultraestructura EZ: bloque (0), cristales finos (1).

8. Proporciones mamilas: más anchas que altas (0), más altas que anchas (1).

9. Sistema de canales: simple (0), bifurcado (1), ramificado (2).

10. Patrón poros: aislados (0), agrupados (1), aepyornitoide (2), cubiertos (3).

11. Ornamentación: presente (1), ausente (0). 